\title{
Evaluación de aptitudes sobresalientes con la SAGES-2 en escolares de tres contextos educativos de Morelos y Sonora
}

Doris Castellanos Simons, ${ }^{1}$ Aldo Mauricio Ferrari Belmont ${ }^{1}$

y Aldo Bazán Ramírez ${ }^{1}$

\section{Introducción}

Uno de los retos educativos en la actualidad es la atención a los alumnos con aptitudes sobresalientes y su detección en el contexto escolar, para lo cual se han desarrollado múltiples procedimientos formales e informales (SEP, 2006; Valadez, 2006). A pesar de la polémica que ha suscitado en las últimas décadas el uso de instrumentos estandarizados para la identificación de los alumnos con un alto potencial en el terreno académico, muchos autores han defendido la utilidad de este tipo de evaluaciones para obtener indicadores iniciales que permitan realizar posteriormente otro tipo de diagnósticos más profundos y multidimensionales (Valadez, 2006; Valdés y Vera, 2012). La aptitud intelectual sobresaliente, Ilamada talento académico cuando cristaliza en productos actuales en áreas curriculares concretas, puede ser comprendida como la integración de un conjunto específico de habilidades y competencias que configuran un perfil

1 Universidad Autónoma del Estado de Morelos. 
particular, susceptible de presentarse como capacidad intelectual general, o de diferenciarse de acuerdo con las disciplinas o áreas académicas; se expresa en un alto potencial de aprendizaje que hace que el estudiante destaque en un contexto educativo y en un grupo etáreo determinado (SEP, 2006); sin embargo, puede suceder que estos alumnos muestren un logro académico por debajo de su verdadero potencial en virtud de factores diversos (personales, del contexto familiar y cultural y, en general, por la ausencia de experiencias estimulantes en su ambiente cercano).

Contar con instrumentos de medición susceptibles de aplicarse en gran escala en las escuelas para ser complementados posteriormente con otras formas de evaluación que den cuenta de la naturaleza del potencial intelectual de los alumnos, se convierte entonces en una premisa y un factor esencial para poder llevar a cabo intervenciones educativas enriquecedoras.

Una de las pruebas desarrolladas con estos fines es la SAGES-2 (Screening Assessment for Gifted Elementary and Middle School Students, $2^{\mathrm{a}}$ edición) de Johnsen y Corn (2003), también validada en México, conocida como Evaluación Inicial para Estudiantes con Aptitudes Sobresalientes. Fue diseñada para identificar: "a los potenciales estudiantes con aptitudes sobresalientes" (Johnsen y Corn, 2003: 17) tanto en inteligencia (vista como aptitud intelectual general o habilidades de razonamiento) como en aprovechamiento en las áreas curriculares de Matemáticas-Ciencias Naturales y de Lengua y Literatura-Ciencias Sociales. La importancia de la misma radicaría, precisamente, en que, según Valdés y Vera (2012), evaluar aspectos relacionados con las aptitudes no circunscribe la detección solamente a aquellos con alto logro en contenidos escolares, en otras palabras, evalúa a la vez, "inteligencia y aprovechamiento" (p. 111) y permite identificar cómo examinar las fortalezas y debilidades del razonamiento y de la competencia escolar de los alumnos.

La SAGES-2, para su versión 4-S (de cuarto de primaria a tercero de secundaria), fue validada en una muestra de 932 alumnos de la Ciudad de México y de Yucatán (Sánchez y Acle, 2003, como se citó en Valdés y Vera, 2012), con un total de 
142 alumnos de sexto de primaria. Al realizar un examen de las propiedades psicométricas del instrumento, los investigadores mexicanos encontraron que en consistencia interna, SAGES-2 4-S obtuvo coeficientes de Alfa de Cronbach de .61 para Matemáticas/Ciencias Naturales; .45 para Lengua y Literatura/Ciencias Sociales y de 0.48 para Razonamiento, con lo cual se recomendó realizar estudios posteriores para estudiar más a fondo estos resultados y la confiabilidad del instrumento. Para examinar la validez concurrente de la prueba, los autores utilizaron las subescalas de la Escala Wechsler de Inteligencia para niños, el WISC-RM, encontrándose 13 correlaciones significativas (de las 42 posibles combinaciones) entre las tres subescalas del SAGES-2 4-S y las subescalas de wisc. Finalmente, los autores también manejaron el análisis de reactivos, la conversión de puntajes brutos a cocientes y de éstos a percentiles, para estandarizar la prueba en las distintas edades de los aplicantes, aunque reconocieron que la muestra utilizada podía resultar limitada en número, lo cual podría estar afectando los valores de consistencia interna al igual que la representatividad de la misma.

El objetivo del presente trabajo fue obtener indicadores de confiabilidad y de validez concurrente para esta prueba, a partir de su aplicación a una muestra de alumnos que cursaban el último trimestre de sexto grado y el primero de secundaria, de escuelas urbanas y rurales en dos entidades federativas: Morelos y Sonora. El estudio formó parte de una investigación más amplia que se desarrolla por la Universidad Autónoma del Estado de Morelos (UAEM) y el Instituto Tecnológico Superior de Sonora (ITSON).

En este caso, las condiciones sociodemográficas del área así como el nivel socioeconómico de la población asistente a las escuelas en cuestión, fueron considerados como indicadores de modalidades que configuran contextos socioeducativos con diferente capital cultural escolar (Backhoff, 2011; Bazán, Sánchez y Castañeda, 2007). 


\section{Método}

\section{Participantes}

La muestra total estuvo conformada por 916 alumnos con edad promedio de 11.9 años, $56.2 \%$ de Morelos y $43.8 \%$ de Sonora. De ellos, $31.4 \%$ pertenecía a escuelas privadas; $34.9 \%$, a escuelas públicas urbanas y $33.6 \%$, a escuelas rurales indígenas, distribuidos entre 19 escuelas en ambas entidades; 52.3\% eran hombres y $47.7 \%$, mujeres.

\section{Material}

Como se señaló, se utilizó la prueba SAGES-2-4S ( $2^{a}$ edición) de Johnsen y Corn (2003) que evalúa la capacidad de razonamiento y conocimientos generales en niños y adolescentes e identifica las aptitudes sobresalientes potenciales en la población escolar. La prueba proporciona puntuaciones directas, cocientes y percentiles. Consta de tres subescalas: 1) Matemáticas/Ciencias Naturales, cuyos reactivos se vinculan con dos áreas académicas de naturaleza lógica-técnica, 2) Lengua y Literatura/Ciencias Sociales, de naturaleza lingüística, y 3) Razonamiento, que mide la capacidad para percibir relaciones y resolver problemas a través de tareas en forma de analogías y reconocimiento de patrones. Las dos primeras escalas comprenden 30 reactivos cada una, con formato de opción múltiple (5 posibles respuestas, 1 correcta), mientras que la última tiene 35 reactivos.

\section{Procedimiento}

Después de obtener la autorización de la escuela y de los padres de los alumnos, se aplicó el SAGES-2 en dos sesiones diferentes; en la primera, los alumnos completaron las subescalas de Matemáticas y Ciencias Naturales y Razonamiento; en la segunda, completaron Lengua, Literatura y Humanidades, con un tiempo máximo de 40 minutos en cada escala. 
En cuanto al análisis y procesamiento de los datos, se buscaron, con ayuda del programa spss versión 22, los indicadores de confiabilidad y validez; para el caso de confiabilidad se utilizó el cálculo del Alfa de Cronbach y para el análisis de validez concurrente, las correlaciones entre las medidas de dos instrumentos para la evaluación de competencias basados en el Plan de Estudio de la Educación Básica 2009 de la SEP, en Matemáticas y Español de sexto grado. Ambos instrumentos fueron construidos y previamente validados por Bazán, Barrera y Vega (2013), y por Ferrari (2014). Asimismo, se analizaron las estadísticas descriptivas para poder examinar la tendencia central y distribución de las escalas, en sus valores cociente y percentil.

\section{Resultados}

Se obtuvo un alfa de .899 utilizando los 95 ítems de la prueba; .762 para la subescala de Matemáticas/Ciencias Naturales; .67 para Lengua y Literatura/Ciencias Sociales, y de .861 para Razonamiento, valores muy superiores a los encontrados por los autores mexicanos reportados en Johnsen y Corn (2003). Igualmente, se realizaron los análisis de confiabilidad para todas las submuestras o subgrupos, sin encontrarse diferencias relevantes entre entidades federativas; sólo se encontró una reducción de más de .15 unidades del coeficiente de Cronbach para la muestra urbana de Sonora (alfa $=0.598$ ) en la subescala de Matemáticas/Ciencias Naturales y para la subescala de Lengua y Literatura/Ciencias Sociales en las submuestras: rural (0.415), Morelos urbano (0.510), Morelos rural (0.421) y Sonora rural (0.405). Esto estaría indicando que la subescala de Lengua y Literatura/Ciencias Sociales muestra la más baja consistencia interna, sobre todo en contextos de escuelas públicas rurales-indígena y escuelas públicas urbanas.

Para el examen de la distribución de las puntuaciones cocientes, se tomó como parámetro los valores esperados por los autores: media de 100 y desviación estándar de 15, encontrándose una diferencia de -2.81 para el valor de la media, con 
una diferencia de +2.49 en desviación estándar para el caso de la subescala de Matemáticas/Ciencias Naturales; de -8.68 y +2.714 para Lengua y Literatura/ Ciencias Sociales y de -1.79 y 3.288 para el caso de Razonamiento. Los casos que más se alejan de lo deseable se encuentran en el contexto rural, donde la diferencia de valores de las medias obtenidas respecto a las deseadas para Matemáticas, Lengua y Razonamiento fue de $-9.49,-17.07$ y -10.81 , respectivamente, aumentando estas diferencias en el contexto rural sonorense.

Para la validez concurrente se obtuvieron correlaciones significativas $(p<0.01)$ y positivas para todas las competencias medidas en los instrumentos de Español y Matemáticas, cuyas escalas son, para Español: Producción de Textos, Comprensión Lectora, y para Matemáticas: Comunicar Información Matemática, Validar Procedimientos y Resultados, Resolver Problemas de Manera Autónoma y Manejar Técnicas Eficientemente. Esto muestra que las escalas del SAGES-2-4-S tienen capacidad predictiva respecto a los resultados en evaluaciones de los contenidos curriculares establecidos en 2009 por la SEP.

\section{Discusión}

Puede decirse que, contrariamente a lo obtenido por Sánchez y Acle (2003), el SAGES 4-S presenta buen grado de confiabilidad en dos de sus tres subescalas: Matemáticas/Ciencias Naturales y Razonamiento, siendo la subescala de Lengua y Literatura/ Ciencias Sociales la que presenta más bajos niveles de confiabilidad.

También se destaca que sería necesario modificar los valores de los cocientes para centrarlos en el valor 100; asimismo, se requeriría una escala de cocientes específica para contextos rurales, ya que con la tabla de probabilidad de aptitudes sobresalientes del instrumento original sería difícil identificar, a través del cociente obtenido por el alumno, valores que se reflejen como probabilidad alta del potencial sobresaliente, lo que denotaría, en última instancia, un alto sesgo sociocultural. En cuanto a va- 
lidez concurrente, se encontró que el SAGES-2 4-S tiene valores de correlación altos con las pruebas de logro académico específicos del Plan de Estudios 2009 para Matemáticas y Español de sexto grado de primaria, a pesar de no estar basado en éste.

Dado que los modelos actuales sobre las aptitudes sobresalientes insisten en el papel decisivo de los factores socioculturales y contextuales en el desarrollo de las potencialidades de niños, adolescentes y jóvenes, es necesario reflexionar sobre estos resultados a la luz de la búsqueda de abordajes sistémicos que den cuenta de la naturaleza multifactorial y compleja de estos fenómenos. Esto apunta a la necesidad de tomar en consideración los aspectos de la diversidad cultural y de validez ecológica de las evaluaciones para llegar a resultados en la investigación psicoeducativa.

\section{Referencias}

Backhoff, E. (2011). La inequidad educativa en México. Profesorado. Revista del currículum y formación del profesorado, 15(3), 88-102.

Bazán, A., Sánchez, B. y Castañeda, S. (2007). Structural relationship Among Parents' Support, Parents' Educational Level, Characteristics of the Teacher and Child's Achievement in Reading and Writing. Revista Mexicana de Investigación Educativa, 3, 701-729.

Bazán, A., Barrrera, D. y Vega, N. (2013). Validación de constructos de competencias de lectura y producción de textos en los inicios de la generalización de la reforma en la Primaria Mexicana. Revista Electrónica Iberoamericana sobre Calidad, Eficacia y Cambio en la Educación, 11(4), 61-76.

Ferrari, A.M. (2014). Validación de instrumentos de evaluación por competencias en Matemáticas y Español en sexto grado de primaria en Morelos y Sonora. Tesis de Licenciatura en Psicología (inédita). Universidad Autónoma del Estado de Morelos, México. 
Johnsen, S.K. y Corn, A.L. (2003). SAGES-2. Evaluación Inicial para Estudiantes con aptitudes sobresalientes (Educación Primaria y Secundaria): Manual. México: Manual Moderno.

Sánchez, P. y Acle, G. (2003). Validación y normas para la población del SAGES-2 mexicana. México: UNAM.

Secretaría de Educación Pública. (2006). Propuesta de intervención: atención educativa a alumnos y alumnas con aptitudes sobresalientes. México.

Valadez, M.D. (2006). Identificación y evaluación de niños superdotados y talentosos. En D. Valadez; J. Betancourt, y M.A. Zavala (Coords.), Alumnos superdotados y talentosos (5380). México: Manual Moderno.

Valdés, A.A. y Vera, J.A. (2012). Estudiantes intelectualmente sobresalientes. México: Pearson Educación. 